\title{
Les Rois mages, images du pouvoir des rois en Occident $\left(\mathrm{XII}^{\mathrm{e}}-\mathrm{XVI}^{\mathrm{e}}\right.$ siècle)
}

Thèse dirigée par Pierre Monnet (EHESS) et Daniel Russo (université de Bourgogne). Soutenue à l'EHESS le 5 décembre 2016

\section{Doina Elena Craciun}

\section{(2) OpenEdition}

12 Journals

\section{Édition électronique}

URL : https://journals.openedition.org/cem/14683

DOI : $10.4000 /$ cem. 14683

ISSN : 1954-3093

Éditeur

Centre d'études médiévales Saint-Germain d'Auxerre

Référence électronique

Doina Elena Craciun, "Les Rois mages, images du pouvoir des rois en Occident ( $x \|^{\mathrm{e}}-\left.\mathrm{xv}\right|^{\mathrm{e}}$ siècle)", Bulletin du centre d'études médiévales d'Auxerre | BUCEMA [En ligne], 21.1 | 2017, mis en ligne le 18 septembre 2017, consulté le 22 septembre 2022. URL : http://journals.openedition.org/cem/14683 ; DOI : https://doi.org/10.4000/cem.14683

Ce document a été généré automatiquement le 22 septembre 2022.

\section{cc) (i) (2)}

Creative Commons - Attribution - Pas d'Utilisation Commerciale - Partage dans les Mêmes Conditions 4.0 International - CC BY-NC-SA 4.0

https://creativecommons.org/licenses/by-nc-sa/4.0/ 


\section{Les Rois mages, images du pouvoir des rois en Occident ( $\mathrm{XII}^{\mathrm{e}}-\mathrm{XVI}^{\mathrm{e}}$ siècle)}

Thèse dirigée par Pierre Monnet (EHESS) et Daniel Russo (université de Bourgogne). Soutenue à l'EHESS le 5 décembre 2016

\section{Doina Elena Craciun}

1 Cette étude porte sur l'association entre le roi médiéval et les Rois mages en Occident (XII $-\mathrm{XVI}^{\mathrm{e}}$ siècle). L'assimilation des rois historiques à ces figures bibliques est visible dans des sources très diverses allant des documents écrits, qui formulent explicitement cette analogie, aux indices fournis par des cérémonies royales couronnements le 6 janvier, offrandes royales d'or, d'encens et de myrrhe le jour de l'Épiphanie, etc. - ou des images où un roi est représenté sous les traits d'un Roi mage.

2 Ce phénomène a été identifié par différents chercheurs, mais un recensement de l'ensemble de sources et une analyse approfondie de cette pratique n'a pas encore été réalisée ${ }^{1}$. L'identification des rois médiévaux avec les Rois mages a intéressé, notamment,

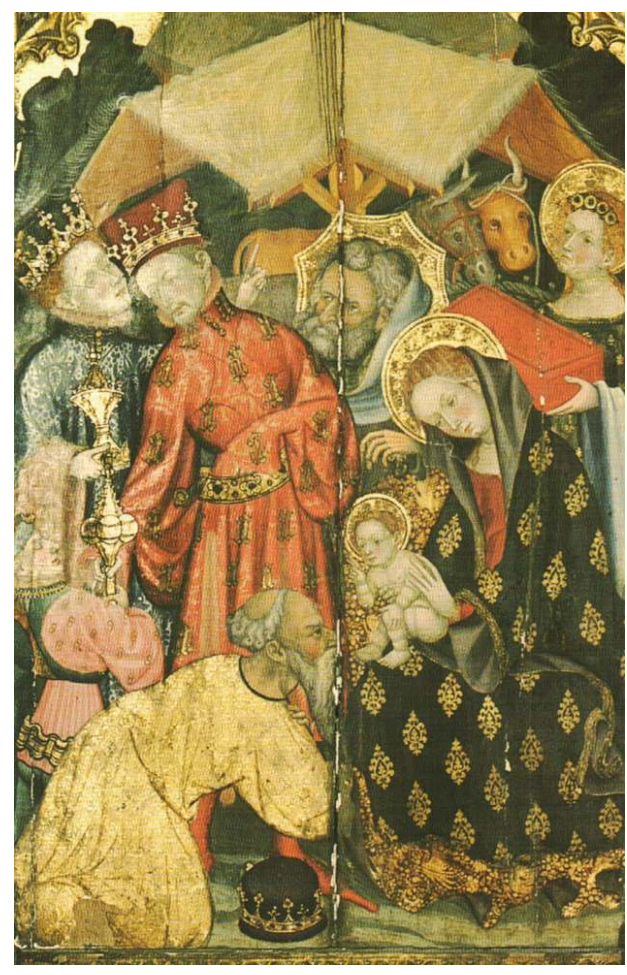
l'historiographie allemande ${ }^{2}$ et, à juste titre, car c'est dans l'Empire romain-germanique que le phénomène connaît la plus grande diffusion, autant dans les textes que dans les images. La moitié des sources que nous avons recensées, en effet, concerne le roi des Romains. Ce développement sans précédent est dû au fait que les reliques des Rois mages sont conservées depuis 1164 à 
Cologne, ville qui entretient tout au long du Moyen Âge des liens forts avec le roi des Romains. Ces liens sont tout d'abord déterminés par le rôle politique de l'archevêque de Cologne, prince électeur, mais aussi figure centrale dans la cérémonie du couronnement des rois des Romains. Dans ce contexte, une relation très étroite est forgée entre le sacre accompli à Aix-la-Chapelle et les Rois mages, dont les corps sont conservés quelques kilomètres plus loin. Ainsi, dès le xIV siècle la messe qui accompagne la cérémonie du couronnement du roi des Romains est celle de l'Épiphanie, alors que, à partir de la seconde moitié du xiII siècle, le sacre est systématiquement suivi d'un voyage à Cologne et d'une visite des reliques des Mages. Mais le cas colonais, auquel cette étude consacre un chapitre fondamental, n'est que l'un des sujets abordés ici.

3 Il nous a paru essentiel de suivre le développement de l'association entre le roi médiéval et les Rois mages dans d'autres contextes, dans des sources produites loin de la ville de Cologne, autant sur le territoire de l'Empire que dans d'autres royaumes. La démarche ne pouvait être satisfaisante qu'en considérant l'ensemble de sources, sur toute la période où le phénomène apparaît, à savoir entre le $\mathrm{XII}^{\mathrm{e}}$ et le $\mathrm{XVI}^{\mathrm{e}}$ siècle. En outre, il nous a paru évident que la signification politique que cette association acquiert à Cologne est différente de celle dont elle est dotée dans d'autres contextes. Cette thèse analyse donc l'assimilation des rois aux Rois mages comme un phénomène à racines multiples, déterminé par des facteurs différents que nous cherchons à identifier.

4 L'un des objectifs de l'étude est de dresser le tableau complet des sources qui créent cette analogie, de les ordonner par royaume et chronologiquement. Nous suivons ainsi l'évolution du phénomène dans chaque espace, mais nous observons aussi son développement à l'échelle de l'Occident médiéval. Immanquablement, l'une des questions qui nous préoccupent ici est celle des possibles transferts des idées et des pratiques d'un royaume à l'autre.

5 En outre, nous prêtons une attention particulière à la question des commanditaires et des auteurs qui créent l'association, ainsi qu'aux significations qu'ils lui confèrent. Sans aucun doute, l'assimilation des rois aux Rois mages est une expression des conceptions de ces acteurs sur la royauté médiévale. Nous cherchons donc à comprendre quelles caractéristiques du roi sont exprimées par ce biais et quelles idées sur le pouvoir royal, ses prérogatives et ses limites, sont transmises par cette analogie.

6 Le recensement de l'ensemble de documents nous a permis d'observer l'évolution du phénomène à l'échelle occidentale. Il apparaît très peu au XII ${ }^{\mathrm{e}}$ siècle, connaît une diffusion plus ample au XIII et un apogée aux XIV et $\mathrm{XV}^{\mathrm{e}}$ siècles. Le nombre de sources où cette association est manifeste baisse $\mathrm{au} \mathrm{xvI} \mathrm{e}^{\mathrm{e}}$ siècle, cette période enregistrant les derniers documents qui créent l'assimilation d'un roi à un Roi mage. Malgré cette évolution générale, chaque royaume suit son propre rythme quant à l'usage de cette analogie. Elle apparaît à des moments différents et la durée de l'attachement à cette association est variable pour chaque espace.

7 L'une des explications possibles pour cette fluctuation réside peut-être dans le fait que certains royaumes s'approprient du thème uniquement sous l'influence d'autres pays ou de certains rois. Par exemple, la Bohême fait usage de l'association entre son roi et les Rois mages uniquement durant le règne de Charles $\mathrm{I}^{\mathrm{er}}$ de Luxembourg (1347-1376) et de son fils Wenceslas IV (1376-1419), tous les deux également rois des Romains portant respectivement le titre de Charles IV et Wenceslas $I^{\text {er }}$. Il est donc possible que le thème soit transféré en Bohême depuis l'Empire romain-germanique pendant le règne 
de Charles IV, roi qui se montre particulièrement concerné par ce rapprochement. Malheureusement, l'état de ce type de transfert peut seulement être supposé, considérant les liens entre deux royaumes pendant une période précise. En réalité, aucun document ne permet d'établir avec certitude les conditions dans lesquelles l'association entre roi et Rois mages naît et perdure dans un certain espace. Et cela d'autant plus qu'elle est créée par des auteurs et des commanditaires provenant de milieux très divers.

8 Le recensement des sources montre, en effet, que le roi et son entourage proche ne constituent qu'une partie de ceux qui conçoivent cette association. Elle est utilisée, en outre, autant par des papes, archevêques, évêques, prédicateurs, abbés ou visionnaires, que par des personnages laïques, patriciens, nobles ou bourgeois. L'analyse de nombreux documents et du contexte de leur production a montré que tous ces acteurs créent l'association entre leur roi et les Rois mages dans des conditions différentes et donnent à cette analogie des significations variées, en fonction de leurs propres conceptions du pouvoir royal. L'étude signale que ces conceptions sont loin d'être homogènes et varient en fonction de la position de chacun de ces acteurs et des rapports qu'il entretient avec le roi assimilé aux Rois mages.

Cette analogie est tout d'abord favorable au roi, car elle permet de souligner son statut particulier entre laïc et clerc et certains pouvoirs et vertus fondamentales pour la royauté médiévale. La perception des Rois mages en tant que rois et prêtres est sans aucun doute un élément intéressant pour revendiquer un statut similaire pour le roi médiéval, sujet qui reste cependant très controversé dans la société médiévale. Certes, le roi médiéval n'est plus un laïc après l'onction, mais ne peut pas prétendre à un vrai statut de clerc. Cette revendication est cependant très présente chez certains rois et elle coïncide avec des initiatives royales d'identification avec l'un des Rois mages. En outre, cette association souligne également les pouvoirs thaumaturgiques de certaines figures couronnées.

Si, pour la France, il est impossible d'établir un lien entre ces pouvoirs du roi et les Rois mages, le rapprochement est très évident pour le roi d'Angleterre. Ce dernier guérit non seulement des écrouelles, mais aussi de l'épilepsie, par un rituel spécifique incluant des anneaux (cramp rings), attesté à partir du XIv siècle. Or, avant que ce rituel soit performé par le roi, c'est le nom des Rois mages gravé sur des anneaux qui était considéré comme remède contre l'épilepsie. $\mathrm{Au} \mathrm{XIV}^{\mathrm{e}}$ siècle, alors qu'apparaissent de nombreuses associations entre le roi d'Angleterre et les Rois mages, s'accomplit également une sorte de transfert du pouvoir thaumaturgique de ces figures bibliques vers le roi. Les anneaux deviennent des objets guérisseurs, non plus en y gravant le nom des Rois mages, mais parce qu'ils sont faits de l'offrande apportée par le roi le Vendredi saint.

11 En outre, dans toute la littérature médiévale, les Mages sont présentés comme des personnages très vertueux, les premiers pèlerins, chrétiens modèles, charitables, généreux et sages. Il est intéressant de constater que toutes les qualités qui leur sont attribuées sont également celles que les «miroirs des princes» exigent du roi médiéval ${ }^{3}$. Associer roi et Rois mages devient ainsi un moyen pour montrer un souverain pèlerin ou qui fait la croisade, possesseur de toutes ces vertus. Et les bénéfices de cette association vont encore au-delà de ces attentes.

12 Certes, les «miroirs des princes" - dont les auteurs sont principalement des ecclésiastiques - promeuvent un roi sage, mais dans la conception de l'Église le roi ne 
peut pas accéder à la sagesse sans l'intermédiaire des clercs, les seuls qui peuvent comprendre les vraies significations des Écritures ${ }^{4}$. Les Rois mages sont justement l'exemple contraire. Leur sagesse n'est pas acquise grâce à une éducation orientée par les clercs, ils sont les successeurs de Balaam, figure biblique qui communique directement avec Dieu. Ainsi, en créant l'identification entre un roi et les Mages, les auteurs médiévaux mettent en évidence un souverain d'une extraordinaire sagesse, qui n'a pas besoin de l'intermédiaire de l'Église pour accéder à la connaissance.

Cette identification sert aussi à légitimer l'intérêt accru pour l'astrologie, ressenti à certaines cours royales à partir du XIII siècle. Si l'astrologie reste tout au long du Moyen Âge une science souvent critiquée par l'Église, elle est essentielle dans l'histoire des Rois mages : c'est grâce à leurs connaissances dans ce domaine qu'ils arrivent à comprendre la signification de l'étoile et à la suivre jusqu'au lieu de naissance du Christ $^{5}$. Par l'assimilation aux Rois mages, les rois cherchent à s'ériger en bons astrologues et à éliminer toute suspicion qui aurait pu persister concernant leur intérêt pour cette science. La sagesse des Mages et leurs connaissances dans le domaine de l'astrologie semblent donc fonctionner comme instrument pour les rois à l'encontre des prétentions de l'Église de contrôler l'accès à la connaissance et de critiquer l'intérêt des rois pour l'astrologie.

Cette étude montre, en outre, que l'assimilation à un Roi mage n'est pas utile au roi seulement dans ses rapports à l'Église, mais aussi dans ses relations aux autres sujets. Ce rôle confère au roi une image de souverain charitable, importante notamment à la fin $d u$ Moyen Âge quand ses tentatives de généraliser l'impôt permanent sont hautement critiquées. Montré dans une scène d'Adoration des Mages, sur le point de faire une offrande (souvent de l'or) à l'Enfant, le roi passe pour un souverain qui redistribue l'impôt qu'il a collecté de ses sujets. Dans ces conditions, la représentation du roi en Roi mage adoucit l'acte d'imposition.

En faveur du roi joue également le nombre des Mages, qui donne la possibilité d'imaginer non seulement un, mais jusqu'à trois rois historiques dans la même scène biblique. Les trois figures sont en outre conçues comme trois hommes d'âges distincts, ce qui incite les auteurs à faire des Rois mages une image des différents rois qui se sont succédé sur un trône. Ce type de texte ou d'image est rarement utilisé pour légitimer la position d'un roi, mais il fonctionne dans la plupart des cas pour glorifier une dynastie, autant dans les monarchies héréditaires que dans celles électives.

Enfin, penser des rois de royaumes différents sous les traits des Rois mages dans une même scène apparaît comme une stratégie pour illustrer des rapports de pouvoir entre plusieurs rois. En effet, pour tous les documents de ce type examinés dans la thèse, la manière dont l'un des Rois mages est mis en avant par rapport aux deux autres reflète des rapports de pouvoir réels entre les rois concernés, au moment où le document a été rédigé ou peint. L'assimilation des rois aux Rois mages devient ainsi une stratégie pour souligner la prééminence d'un roi sur ses pairs.

Il est intéressant de constater que les mêmes stratégies utilisées pour marquer la supériorité d'un roi fonctionnent également pour donner à un roi une position d'infériorité par rapport à un autre personnage représenté. Dans certaines images, le rôle du Mage qui est mis en avant est joué par un patricien ou un bourgeois, alors que le roi occupe la place d'un Mage plus humble, moins bien visible donc moins important. L'analyse de ces documents a apporté une conclusion fondamentale pour cette étude : l'assimilation d'un roi à un Roi mage n'est pas toujours créée en faveur du souverain, 
mais elle peut servir les intérêts d'autres personnages contemporains qui se positionnent ainsi en concurrence avec le pouvoir royal.

Le rapport entre la Vierge à l'Enfant et les Mages dans la scène de l'Adoration des Mages est parfois utilisé dans ce même but. De nombreuses études ont montré comment l'Église - entendue comme institution et représentée par les autorités ecclésiastiques est identifiée au Moyen Âge à la Vierge à l'Enfant ${ }^{6}$. Examinant un nombre de sources en fonction de ces observations, nous avons montré que certaines Adorations des Mages avec des crypto-portraits royaux étaient conçues comme des représentations de la relation entre un pape ou un archevêque - représenté sous les traits de la Vierge à l'Enfant - et un roi - dans le rôle d'un Mage. Pour certains commanditaires, l'analogie entre rois médiévaux et Rois mages est donc utilisée pour mettre en image la relation entre l'autorité ecclésiastique et le pouvoir royal, le dernier étant dominé par la première.

Dans son ensemble, cette thèse montre que la richesse du portrait des Rois mages et les relations qu'ils entretiennent entre eux et avec les autres personnages - notamment dans la scène de l'Adoration des Mages - donnent à ces figures une extraordinaire plasticité. Ainsi, par la substitution d'un ou des trois Mages avec des rois médiévaux dans une scène de l'Adoration des Mages se transmettent les conceptions les plus diverses concernant le pouvoir royal. Cette scène donne la possibilité de montrer non seulement un roi-prêtre, thaumaturge, pieux, charitable, pèlerin ou qui fait la croisade, sage, astrologue, puissant et légitime, mais aussi un roi soumis, ou dont le pouvoir est inférieur à l'autorité d'un évêque, du pape ou d'un laïc.

Reçu : 27 février 2017 - Accepté : 3 mai 2017

\section{NOTES}

1. Les travaux antérieurs se concentrent principalement sur un type de source ou analysent seulement des documents isolés, mentionnant au passage la diffusion plus large du phénomène.

2. Les études les plus détaillées du sujet sont H. STEHKÄMPER, « Könige und Heilige Drei Könige », in R. BUDDE RAINER (dir.), Die Heiligen Drei Könige, Darstellung und Verehrung : Katalog zur Ausstellung des Wallraf-Richartz-Museums in der Josef-Haubrich-Kunsthalle Köln, Cologne, 1982, p. 37-50 ; A. GRAF, Herrscherporträts in Dreikönigsdarstellungen im 15. Jahrhundert, thèse soutenue à l'université de Salzbourg, 1988; G.SCHWEDLER, «Ritualinnovation. Zur Gestaltung politisch-liturgischer Zeremonien im Spätmittelalter am Beispiel der Pariser Dreikönigsmesse im Jahre 1378 », in B. DÜCKER et G. SCHWEDLER (dir.), Das Ursprüngliche und das Neue. Zur Dynamik ritueller Prozesse in Geschichte und Gegenwart, Berlin, 2008, p. 145-206.

3. Sur les «miroirs des princes", voir notamment J. HRYNEN, L'empire du roi: idées et croyances politiques en France, XIII ${ }^{e}$-XV siècle, Paris, 1993 et I. P. BEJCZY et C. J. NEDERMAN (dir.), Princely Virtues in the Middle Ages, 1200-1500, Turnhout, 2007.

4. Au sujet de cette mainmise des clercs sur le savoir et la manière dont elle devient un lieu où se manifeste la concurrence entre le pouvoir royal et l'Église, voir notamment P. BUC, L'ambiguité du Livre : prince, pouvoir et peuple dans les commentaires de la Bible au Moyen Âge, Paris, 1994, p. 176-205. 5. V. I. J. FLINT, The Rise of Magic in Early Medieval Europe, Princeton, 1991, p. 370 sq. 
6. À ce sujet, voir, par exemple, l'étude de M-L. THÉREL, Le Triomphe de la Vierge-Église : à l'origine du décor du portail occidental de Notre-Dame de Senlis, sources historiques, littéraires et iconographiques, Paris, 1984, p. 78-202.

\section{INDEX}

Mots-clés : Rois mages, pouvoir royal, gouvernement, Occident, iconographie, légitimation, image, représentation

\section{AUTEUR}

\section{DOINA ELENA CRACIUN}

Docteur de l'EHESS/Centre de recherches historiques (groupe : anthropologie historique du long Moyen Âge occidental) 